\title{
Servikal miyelopati tedavisinde anterior ve posterior yaklaşımların karşılaştırılması
}

\section{Comparison of anterior and posterior approaches in cervical myelopathy treatment}

\author{
Mehmet Aydoğan ${ }^{1}$, Ayhan Kılıç² \\ ${ }^{1}$ Fulya Ortopedi ve Omurga Merkezi, İstanbul \\ ${ }^{2}$ Acıbadem Taksim Hastanesi Ortopedi ve Travmatoloji Kliniği, İstanbul
}

\begin{abstract}
Servikal dejeneratif hastalıkların cerrahi tedavisinde yaklaşım, hastanın patolojisine göre, anterior, posterior veya anterior-posterior (kombine) yöntemler şeklinde değişiklik gösterir. Her bir yaklaşımın avantajları ve dezavantajları mevcuttur. Anterior yaklaşımlar; basit tek seviye disketomi ve füzyon, multi-seviye diskektomi ve füzyon, artroplasti ve korpektomiyi içerir. Posterior yaklaşımlar ise laminektomi, laminoplasti ve laminoforaminotomidir. Cerrahisiz doğal seyir nörolojik kötüleşmeyle sonuçlanabilir. Öte yandan, cerrahi dekompresyon birçok hastada nörolojik iyileşme veya nörolojik durumun korunması ile sonuçlanmaktadır. ${ }^{[1]}$ Multi-seviye servikal miyelopatinin cerrahi tedavisi, temel olarak korpektomi ve posteriordan, laminektomi veya laminoplasti olarak iki yolla yapılır. Ancak, hangi yaklaşımın daha iyi klinik sonuçlar sağladığına dair tartışmalar hala devam etmektedir.
\end{abstract}

Anahtar sözcülkler: servikal disk hastalığı; anterior yaklaşım; posterior yaklaşım
Surgical approach to surgical treatment of cervical degenerative diseases differs according to the patient's pathology as anterior, posterior or anterior-posterior (combined) surgical methods. Each approach has its advantages and disadvantages. Anterior approaches include simple single level discectomy and fusion, multi-level discectomy and fusion, arthroplasty and corpectomy. Posterior approaches include laminectomy, laminoplasty and laminoforaminotomy. Natural course without surgery can result in neurological deterioration. On the other end, surgical decompression results in neurological recovery or preservation of the neurological condition of many patients. Surgical treatment of multi-level cervical myelopathy is mainly performed in two ways: corpectomy, and posterior laminectomy or laminoplasty. However, debate still continues as to which approach provides better clinical outcomes.

Key words: cervical disc dissease; anterior approach; posterior approach

\section{ANTERIOR YAKLAŞIMLAR}

Anterior yaklaşımda hedef, servikal miyelopatinin anteriordan dekompresyonu ve füzyonudur. Tek seviye patolojiler, basit diskektomi+füzyon ya da artroplasti ile tedavi edilebilir. Yine, multi-seviye patolojilerde çoklu seviye disketomi ve füzyon sonuçları da başarılıdır. Şiddetli miyelopati veya OPLL (ossifiye posterior longitudinal ligament) de multi-level korpektomi ve strut greft tekniği ile tedavi edilir. ${ }^{[1,2]}$

\section{DORSAL YAKLAŞIMLAR}

Dorsal yaklaşımda tipik olarak üç temel yöntem mevcuttur: 1) laminektomi, 2) laminektomi ile birlikte füzyon ve 3) laminoplasti. Laminektomi eski bir tekniktir ve miyelopatinin tedavisinde günümüzde nadir kullanılmaktadır. ${ }^{[3]}$ Büyük oranda post-laminektomi kifoza neden olur ve tedavisi oldukça güçlükler içerir. Laminektomi ile birlikte füzyon, dorsal dekompresyon ve stabilizasyonu içerir ve cerrahi sonrası kifoz veya geç instabiliteyi önler. Laminoplasti, yumuşak doku iyileşmesini sağlayıp kifozu minimize etmek için kemik arkın devamlılığını sağlayan ve bir miktar hareketi sürdüren, servikal stenozun tedavisinde en sık kullanılan tartışmalı yöntemlerdendir. ${ }^{[4]}$

\section{YAKLAŞIMIN SEÇiMi}

Genç hastalar her iki yaklaşımı da tolere edebilirler. Yaşı ı şiddetli akciğer rahatsızlığı olan hastalar, kısa süreli posterior yaklaşımlara çok iyi adaydırlar. Benzer şekilde, yaşı ı çok fazla medikal komorbiditesi olan

- Illetişim adresi: Doç. Dr. Mehmet Aydoğan, Dikilitaş Mah. Ayazmadere Cad. Yeşilçimen Sok. No:9/3 Kat: 3, Fulya, Beşiktaş, İstanbul Tel: 0212 - 2276338 e-posta: ortospine@yahoo.com

- Geliș tarihi: 5 Nisan $2017 \quad$ Kabul tarihi: 5 Nisan 2017 
hastalar, tek seanslı üç seviye korpektomi ile strut greft ve posterior enstrümantasyonu tolere edemeyebilirler. Bu hastalarda dorsal bir yaklaşım; laminektomi ve füzyon veya laminoplasti daha iyi tolere edilebilir.

Cerahi öncesi aksiyel boyun ağrısı da cerrahın yaklaşımını etkileyen faktörlerdendir. Artrodez, dejeneratif segmentte hareketi durdurup boyun ağrısını minimize edebilir. Laminoplasti, kanal dekompresyonun yanı sıra, hareketi koruması ve cerrahi sonrası kifozu önlemesiyle 1980 ve 1990 'larda popülarize olmuş bir yöntemdir. 1996'da Hosono[5], laminoplasti hastalarının cerrahi sonrası uzun dönem takibinde, belirgin bir oranda (\%25) boyun ağrısı bildirmiştir. Bir çalışmada, open-door laminoplastide French door tekniğe oranla daha fazla boyun ağrısı olduğunu bildirilmiştir. ${ }^{[6]}$ Cerahi öncesi boyun ağrısı, yaklaşımın seçiminde hala önemli bir faktördür. Masaki, yaşlı laminoplasti ile tedavi ettiği yaşlı hastalarda cerrahi sonuçların kötü olduğunu bildirmiş ${ }^{[7]}$; Shibuya ise tersine, yaşlı hastalarda laminoplastiyi önermiştir. ${ }^{[8]}$

Cunningham, tek segmenti içeren sistemik derlemede, anterior servikal diskektomi ve füzyon, korpektomi, laminektomi ve laminoplasti sonuçlarını karşılaştırmıştır. Bu çalışmada; kanama miktarı, operasyon süresi, cerrahi sonrası komşu segment dejenerasyonu ve komplikasyon oranları, anterior cerrahi segmentte yüksek bulunmuştur. ${ }^{[9]}$

Zhu ve arkadaşları, korpektomi ve laminoplasti ya da laminektomi ile multi-seviye servikal cerrahi yapılan sekiz çalışmayı içeren derlemelerinde, cerrahi travmanın, kanama ve operasyon sürelerinin korpektomi grubunda belirgin ölçüde yüksek olduğunu bildirmişlerdir. ${ }^{[10]}$

Posterior laminektomi ile dekompresyon ve fiksasyon, kısmen basit ve güvenilir, iyi tanımlanmış bir tekniktir; yazarlar, multi-seviye spondilotik miyelopatide başarılı ve etkili bir yöntem olduğunu bildirmişlerdir; kifoz gelişimini engelleyerek uzun dönem iyi bir stabilite sağlamaktadır. ${ }^{[10]}$

Laminoplasti sonrasındaki aksiyel boyun ağrısının kesin nedeni belli değildir. Ağrının potansiyel nedeni, laminoplasti sonrasında servikal hareket kaybı ve posterior ekstansör mekanizmanın zarar görmesi olabilir. C2 ve C7'ye yapışan ekstansör mekanizmanın korunduğu "modifiye laminoplasti" ile, cerrahi sonrası aksiyel ağrı azaltılabilir. ${ }^{[11,12]}$

Wada, füzyon yapılan segment sayısı ile psödoartroz arasında belirgin bir korelasyon olduğunu bildirmiştir. ${ }^{[13]}$ Fraser, 2.682 hastalık bir meta-analizde, anterior plakla füzyon oranının tek seviye disketomide $\% 97,1$, iki seviye diskektomide $\% 94,6$ ve üç seviye diskektomide \%82,5 olarak bildirmiştir. ${ }^{[14]}$ Yine, multi-seviye korpektomi teknik olarak zor ve pseodoartroz oranı yüksektir.

Yaklaşım seçimi kadar önemli olan bir konuda greft seçimidir. Weber, korpektomi yapılan 75 hastada kullanılan farklı greft materyallerini incelemiştir. Bu çalışmada; fibula allogreftini, titanyum mesh kafesi, karbon fiber kafesi, genişleyebilir titanyum kafesi içeren farklı tasarımları karşılaştırmıştır. Ortalama greft kollapsı için; fibular allogreftte $3 \mathrm{~mm}$, revizyon \%25; titanyum mesh kafeste 2,9 mm, revizyon \%11,1; titanyum genişleyebilir kafeste ise $\% 18,8$ revizyon bildirmiştir. Ortalama greft kollapsını karbon fiber kafeslerde $0,7 \mathrm{~mm}$ saptarken, hiçbir hastanın revizyona gitmediğini bildirmiştir. ${ }^{[15]}$

Laminoplasti sonrasında servikal omurgadaki kifotik değişiklikler iyi bilinmektedir. Kimura, kifotik veya $\mathrm{S}$ şeklindeki malaligment'larda laminoplastinin iyi klinik sonuçlar sağlayamadığını ve ilerleyen kifozun nörolojik kötüleşmeye neden olduğunu bildirmiştir. ${ }^{[16]}$ Kifotik postür, spinal kordun anteriorda vertebraya yapışarak vertebral gövde ya da disk tarafından basısına neden olur. Cerahi öncesi fleksiyon-ekstansiyon grafisi, kifozun fikse ya da fleksibl olup olmadığı konusunda bilgi verir. Eğer fleksibl ise, ekstansiyon ile ne kadar korreksiyon elde edilebileceği konusunda fikir edinilebilir. Eğer rezidüel kifoz olursa, bunun anteriordan basıya devam edeceği ve bu nedenle anterior dekompresyonun daha etkili olacağı akılda tutulmalıdır.

Anterior dekompresyon bir veya iki seviyede endikedir ve daha etkili bir kifoz düzeltimi sağlar. Üç veya daha fazla segment etkilenmişse, non-union oranı yüksektir ve posterior yaklaşım önerilir. Eğer laminektomi yapılmışsa, cerrahi sonrası dönemde geç kifozu önlemek için mutlaka stabilizasyon gerekir. Kifozla birlikte multi-seviye patolojilerde, spinal kord osteofitler tarafindan anteriordan gerilir. Bu olgular, anterior yaklaşım kullanılarak anterior gevşetme ile lordozun sağlanmasının yanı sıra, posteriordan dekompresyonla kombine yaklaşım için de uygundur. ${ }^{[17,18]}$

Servikal miyelopatili hastaların bir bölümü, tek başına ne anterior ne de posterior yaklaşımla tedavi edilebilir. Bazı hastalarda, kombine veya $360^{\circ}$ prosedürlere ihtiyaç duyulur. Belirgin osteoporoz, belirgin deformite ve postlaminektomi hastaları, üç veya daha fazla seviye korpektomi ve strut greft yapılan hastalar, kombine yaklaşımlar için adaydırlar (Şekil 1).

Belirgin osteoporozu olan hastalarda, anterior plaklama zayıf kalabilir ve greft-plak ayrışması ile sonuçlanabilir. Beraberinde yapılan posterior enstrümantasyon, greft ve plak çıkmasını engelleyebilir. Belirgin deformite hastalarında, omurga cerrahi öncesi pozisyonuna tekrar dönmek ister. Belirgin kifotik deformite, 

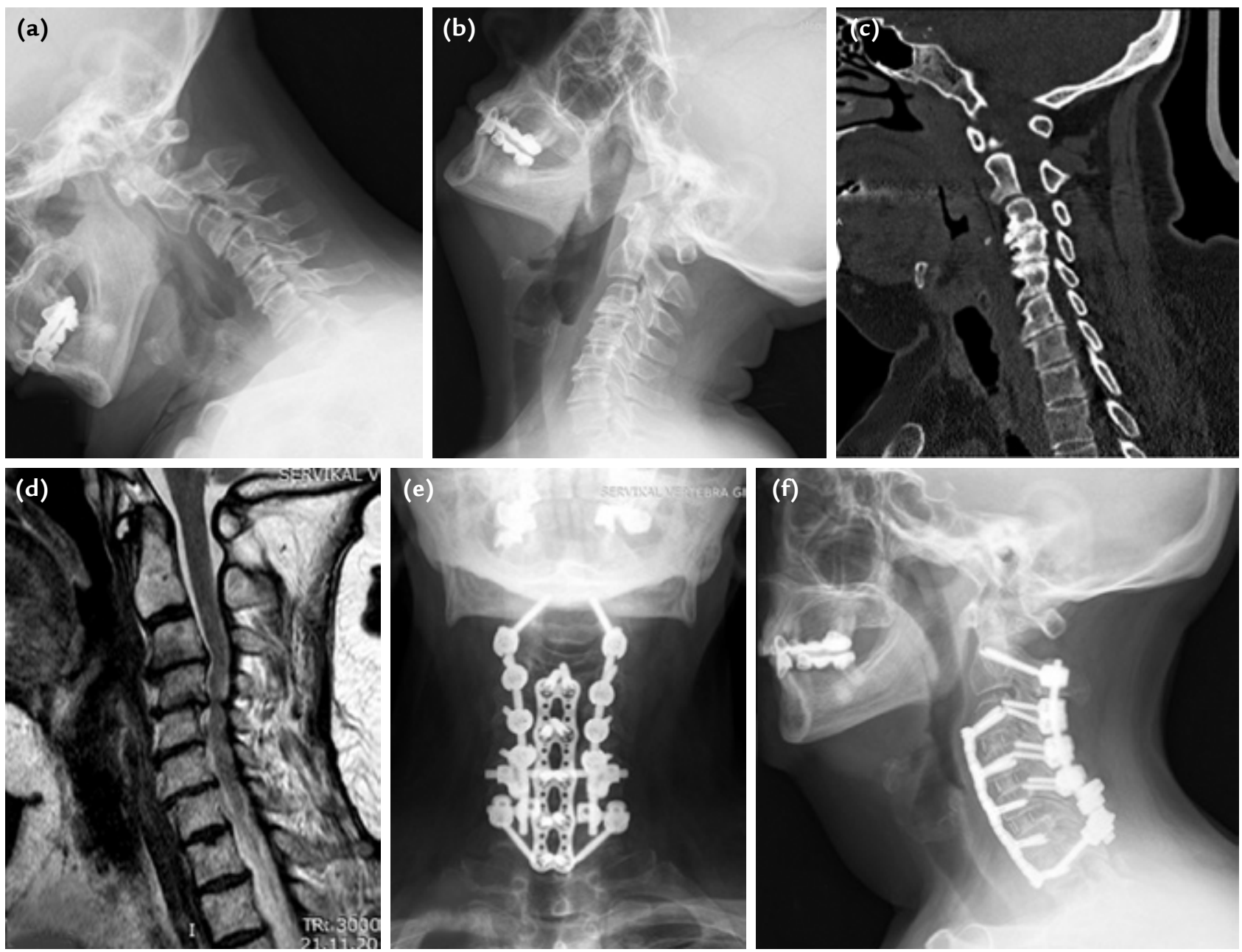

Şekil 1. a-d. Altmış yedi yaşında bayan hasta; yürüme bozukluğu, kollarda yanma ve uyuşukluk mevcut. Hastanın ameliyat öncesi servikal fleksiyon (a), ekstansiyon (b) grafileri; servikal sagittal BT (c) ve servikal sagittal MR (d) kesitleri; kombine cerrahi sonrasında servikal ön-arka (e) ve yan (f) grafileri.

yine belirgin fleksiyon kuvvetlerine neden olur ve bu, rekonstrüksiyon ile karşılanmalıdır. Şiddetli cerrahi öncesi kifoz hastalarında sagittal plan korreksiyonunu sürdürmek için, posteriordan enstrümantasyon ile tansiyon bandı oluşturmak gerekir. Postlaminektomi hastalarında dorsal kemik arkının ve yapışık kasların çıkartılması, servikal omurganın stabilitesinin azalmasına neden olacaktır. Post-laminektomi hastalarında multi-seviye korpektomiye ihtiyaç duyuluyorsa, yüksek oranda posterior enstrümantasyon tavsiye edilir. Riew, dorsal plaksız greft komplikasyonlarını oldukça yüksek bildirmiştir. ${ }^{[19]}$ Vaccaro ve Sasso, üç veya daha fazla seviyede fibula strut greft ve anterior plak yapılan hastalarda, yüksek oranda başarısızlık bildirmişlerdir. ${ }^{[20,21]}$
Sonuç olarak; anterior veya posterior cerrahiyi seçerken, hastanın yaşı, komorbiditeleri, patolojinin yerleşim yeri ve şekli, cerrahi öncesi servikal sagittal dizilim ve cerrahın tecrübesine bağlı faktörler etkili olur. Özellikle belirgin osteoporozu olan, üç veya daha fazla korpektomi yapılan ve belirgin deformitesi olan hastalarda, kombine yaklaşımlar tercih edilmelidir.

\section{KAYNAKLAR}

1. Sugrue PA, McClendon J Jr, Halpin RJ, Liu JC, Koski TR, Ganju A. Surgical management of cervical ossification of the posterior longitudinal ligament: natural history and the role of surgical decompression and stabilization. Neurosurg Focus 2011;30(3):E3. Crossref 
2. Emery SE, Bohlman $\mathrm{HH}$, Bolesta MJ, Jones PK. Anterior cervical decompression and arthrodesis for the treatment of cervical spondylotic myelopathy. Two to seventeen-year follow-up. J Bone Joint Surg Am 1998;80(7):941-51.

3. Anderson PA, Matz PG, Groff MW, Heary RF, Holly LT, Kaiser MG, Mummaneni PV, Ryken TC, Choudhri TF, Vresilovic EJ, Resnick DK; Joint Section on Disorders of the Spine and Peripheral Nerves of the American Association of Neurological Surgeons and Congress of Neurological Surgeons. Laminectomy and fusion for the treatment of cervical degenerative myelopathy. J Neurosurg Spine 2009;11(2):150-6. Crossref

4. Matz PG, Anderson PA, Groff MW, Heary RF, Holly LT, Kaiser MG, Mummaneni PV, Ryken TC, Choudhri TF, Vresilovic EJ, Resnick DK; Joint Section on Disorders of the Spine and Peripheral Nerves of the American Association of Neurological Surgeons and Congress of Neurological Surgeons. Cervical laminoplasty for the treatment of cervical degenerative myelopathy. J Neurosurg Spine 2009;11(2):157-69. Crossref

5. Hosono N, Yonenobu K, Ono K. Neck and shoulder pain after laminoplasty. A noticeable complication. Spine (Phila Pa 1976) 1996;21(17):1969-73.

6. Okada M, Minamide A, Endo T, Yoshida M, Kawakami M, Ando M, Hashizume H, Nakagawa Y, Maio K. A prospective randomized study of clinical outcomes in patients with cervical compressive myelopathy treated with opendoor or French-door laminoplasty. Spine (Phila Pa 1976) 2009;15;34(11):1119-26. Crossref

7. Masaki Y, Yamazaki M, Okawa A, Aramomi M, Hashimoto M, Koda M, Mochizuki M, Moriya $\mathrm{H}$. An analysis of factors causing poor surgical outcome in patients with cervical myelopathy due to ossification of the posterior longitudinal ligament: anterior decompression with spinal fusion versus laminoplasty. J Spinal Disord Tech 2007;20(1):7-13. Crossref

8. Shibuya S, Komatsubara S, Oka S, Kanda Y, Arima N, Yamamoto T. Differences between subtotal corpectomy and laminoplasty for cervical spondylotic myelopathy. Spinal Cord 2010;48(3):214-20. Crossref

9. Cunningham MR, Hershman S, Bendo J. Systematic review of cohort studies comparing surgical treatments for cervical spondylotic myelopathy. Spine (Phila Pa 1976) 2010;35(5):537-43. Crossref

10. Zhu B, Xu Y, Liu X, Liu Z, Dang G. Anterior approach versus posterior approach for the treatment of multilevel cervical spondylotic myelopathy: a systemic review and meta-analysis. Eur Spine J 2013;22(7):1583-93. Crossref
11. Sakaura $H$, Hosono $N$, Mukai $Y$, Fujimori $T$, Iwasaki $M$, Yoshikawa $\mathrm{H}$. Preservation of muscles attached to the $\mathrm{C} 2$ and C7 spinous processes rather than subaxial deep extensors reduces adverse effects after cervical laminoplasty. Spine (Phila Pa 1976) 2010;15;35(16):E782-6. Crossref

12. Kotani $Y$, Abumi $K$, Ito $M$, Sudo $H$, Takahata $M$, Nagahama K, Iwata A, Minami A. Impact of deep extensor musclepreserving approach on clinical outcome of laminoplasty for cervical spondylotic myelopathy: comparative cohort study. Eur Spine J 2012;21(8):1536-44. Crossref

13. Wada E, Suzuki S, Kanazawa A, Matsuoka T, Miyamoto $\mathrm{S}$, Yonenobu K. Subtotal corpectomy versus laminoplasty for multilevel cervical spondylotic myelopathy: a longterm follow-up study over 10 years. Spine (Phila Pa 1976) 2001;26(13):1443-7.

14. Fraser JF, Härtl R. Anterior approaches to fusion of the cervical spine: a metaanalysis of fusion rates. J Neurosurg Spine 2007;6(4):298-303. Crossref

15. Weber MH, Fortin M, Shen J, Tay B, Hu SS, Berven S, Burch S, Chou D, Ames C, Deviren V. Graft Subsidence and Revision Rates Following Anterior Cervical Corpectomy: A Clinical Study Comparing Different Interbody Cages. Clin Spine Surg 2016. [Epub ahead of print] Crossref

16. Kimura I, Shingu $H$, Nasu Y. Long-term follow-up of cervical spondylotic myelopathy treated by canal-expansive laminoplasty. J Bone Joint Surg Br 1995;77(6):956-61.

17. Fehlings MG, Barry S, Kopjar B, Yoon ST, Arnold P, Massicotte EM, Vaccaro A, Brodke DS, Shaffrey C, Smith JS, Woodard E, Banco RJ, Chapman J, Janssen M, Bono C, Sasso R, Dekutoski M, Gokaslan ZL. Anterior versus posterior surgical approaches to treat cervical spondylotic myelopathy: outcomes of the prospective multicenter AOSpine North America CSM Study in 264 patients. Spine (Phila Pa 1976) 2013;38:2247-52.

18. Sun Y, Li L, Zhao J, Gu R. Comparison between anterior approaches and posterior approaches for the treatment of multilevel cervical spondylotic myelopathy: A meta-analysis. Clin Neurol Neurosurg 2015;134:28-36. Crossref

19. Riew KD, Hilibrand AS, Palumbo MA, Bohlman HH. Anterior cervical corpectomy in patients previously managed with a laminectomy: short-term complications. J Bone Joint Surg Am 1999;81(7):950-7.

20. Vaccaro AR, Falatyn SP, Scuderi GJ, Eismont FJ, McGuire RA, Singh K, Garfin SR. Early failure of long segment anterior cervical plate fixation. J Spinal Disord 1998;11(5):410-5.

21. Sasso RC, Ruggiero RA Jr, Reilly TM, Hall PV. Early reconstruction failures after multilevel cervical corpectomy. Spine (Phila Pa 1976) 2003;28(2):140-2. Crossref 